\title{
Smoothing by cubic spline modified applied to solve inverse thermal problem
}

\author{
Leticia Hiromi Kubo $^{1}$. Juliana de Oliveira ${ }^{1}$
}

Received: 22 September 2016 / Accepted: 29 September 2016/ Published online: 24 October 2016

(C) SBMAC - Sociedade Brasileira de Matemática Aplicada e Computacional 2016

\begin{abstract}
This paper presents an alternative to cubic spline regularization and its weighted form applied in solving inverse thermal problems. The inverse heat transfer problems are classified as ill-posed, that is, the solution may become unstable, mainly because they are sensitive to random errors deriving from the input data, necessitating a regularization method to soften these effects. The smoothing technique proposed by cubic spline regularization ensures that the global data tend to be more stable, with fewer data oscillations and dependent on a single arbitrary parameter input. It also shows that the weighted cubic spline is able to enhance filter action. The methods have been implemented in order for the search engine to optimize the choice of parameters and weight and, thus, the smoothing gains more flexibility and accuracy. The simulated and experimental tests confirm that the techniques are effective in reducing the amplified noise by inverse thermal problem presented.
\end{abstract}

Keywords Cubic spline $\cdot$ Regularization $\cdot$ Ponderation $\cdot$ Inverse problem

Mathematics Subject Classification $65 \mathrm{~F} 22 \cdot 65 \mathrm{D} 07 \cdot 65 \mathrm{D} 10 \cdot 80 \mathrm{~A} 23$

\section{Introduction}

Recently, the application and theory of the Inverse Heat Transfer Problems (IHTP) have grown increasingly to be found in almost all branches of science and engineering. Chemical, mechanical, nuclear and aerospace engineers, statisticians, physicists, mathematicians, among others,

Communicated by Cristina Turner.

Juliana de Oliveira

juliana@assis.unesp.br

Leticia Hiromi Kubo

leticiahk@assis.unesp.br

1 Department of Biological Sciences, Faculty of Sciences and Letters of Assis FCLA, University of São Paulo State, UNESP, Av. Dom Antonio 2100, Assis, SP 19806-900, Brazil 
are focused on the subject, even with their different needs and applications (Özisik and Orlande 2000; Kim et al. 2006; Kabala 1997; Park et al. 1999; Kaipio and Fox 2011).

The IHTP are, however, classified as ill-posed. While the notion of a well-posed or "well-designed" mathematical problem came in 1923 with discussions on the Hadamard's work (Hadamard 1923) in which the determining conditions are generally the existence properties, uniqueness and (by implication) stability of solutions (Beck et al. 1985), which the inverse problems do not. This occurs because the mathematical formulation of physical processes in thermal sciences has an unknown amount of constants, such as temperature measurements, heat flow, radiation intensity, among others (Orlande 2011).

Difficulties associated with IHTP occur by bringing solutions that can become unstable as the results originated from measurements with inherent errors in analyses (Özisik and Orlande 2000), mainly because they have input data sensitive to random errors (Orlande 2011), as inserted noise in the experimental data. During decades, it was believed that if any well-posed conditions were disrupted, the problem would either be insoluble or generate meaningless and unpractical results (Orlande 2011). This notion only changed with Tikhonov's regularization technique (Tikhonov and Arsenin 1977), Alifanov's iterative regularization method (Alifanov 1977, 1994) and Beck's sequential function specification technique (Beck et al. 1985).

A successful solution of an inverse problem usually involves reformulating via association with a well-posed problem. In most methods, the solution of IHTP is obtained by least squares method. After 1960s and 1970s, many other methods were formalized in terms of treatment capacity (Orlande 2011). The ideal regularization depends on the selection of the appropriate algorithm and choice of specific parameters for each method, in order to achieve the problem's solution, for example, the best compromise between data recovery and signal smoothing. Techniques such as moving average filtering, simplified least squares method (as Savitzky and Golay), splines, Fast Fourier Transform and wavelet have been used for noise treatment. The main purpose is the application of these methods to improve signal to noise ratio (Jakubowska 2011).

Among the various regularization methods, smoothing by cubic spline presents efficient results because it ensures a global smoothing of data and tends to decrease the probability of data oscillations and increase stability. The regularization by splines is made from spline functions which are obtained when a data set is divided into subintervals. Among the various possible spline function divisions, order three provides good flexibility, being connected by curves with continuous first and second derivatives in the internal points and their four constants allow a good flexibility (Reinsch 1967).

Beyond the standard regularization by cubic spline, it is possible to modify an existing method by adding a weight to intensify the filter action and trying to bring the most promising results. So the aim of this study is to reconstruct as faithfully as possible the monitored temperature of a thermal process, which suffers all the implications of inverse problems and needs a strong regularization technique to soften the noise and delays caused by experimental modeling, and is still efficient to maintain the original feature of the signal. This work presents the efficiency of cubic spline regularization implemented by comparing it with the pre-defined MATLAB ${ }^{\circledR}$ function and also an alternative form of weighted smoothing as described by Green and Silverman (1994).

\section{Derivative of the inverse problem}

The mathematical model of thermal process in this work, aims at reconstructing the temperature, is based on Oliveira et al. (2006), and considers heat accumulation and heat transfer 
between the medium and thermal sensor by convection and radiation-in this case, a thermocouple; thus, the transduction equation:

$$
\mathrm{MC} \frac{\mathrm{d} T}{\mathrm{~d} t}-h A\left(T_{\mathrm{viz}}-T_{\mathrm{s}}\right)-\varepsilon \sigma A\left(T_{\infty}^{4}-T_{\mathrm{s}}^{4}\right)=0
$$

is defined by the encapsulation mass $M(\mathrm{~kg})$ and specific heat $C(\mathrm{~J} / \mathrm{Kg} \mathrm{K})$. The properties of convection and radiation passing through area $A\left(\mathrm{~m}^{2}\right)$, respectively, are defined by $h$ $\left(\mathrm{W} / \mathrm{m}^{2} \mathrm{~K}\right)$, convection coefficient and $\varepsilon$ (dimensionless), emissivity and by the StefanBoltzmann constant $\left(\sigma=5.670 \times 10^{-8} \mathrm{~W} / \mathrm{m}^{2} \mathrm{~K}^{4}\right)$. The heat conduction through the sensor cable has been disregarded due to the negligible wire area.

Assuming that the sensor is immersed in medium, the equation that relates to the real process temperature signal captured by the probe without encapsulation $T_{\text {proc }}$, and the indicated temperature signal captured by the probe with encapsulation $T_{\text {ind }}$, provides a delayed and attenuated signal temperature, which may be written as:

$$
\mathrm{MC} \frac{\mathrm{d} T_{\text {ind }}}{\mathrm{d} t}-h A\left(T_{\text {proc }}-T_{\text {ind }}\right)-\varepsilon \sigma A\left(T_{\infty}^{4}-T_{\text {ind }}^{4}\right)=0
$$

where $T_{\infty}$ is the radiative transfer temperature.

Equation (2) can be expressed dividing both sides by $h A$ and reorganizing the powers of terms $T_{\infty}$ and $T_{\text {ind }}$ as:

$$
\tau \frac{\mathrm{d} T_{\text {ind }}}{\mathrm{d} t}-h A\left(T_{\text {proc }}-T_{\text {ind }}\right)-\gamma\left(T_{\infty}-T_{\text {ind }}\right)=0
$$

where $\tau=\frac{\mathrm{MC}}{h A}$ denotes the sensor's constant of time, that is, the accumulation of heat over the heat transferred by convection caused by the temperature increase and $\gamma \cong \frac{4 \varepsilon \sigma}{h}\left(\frac{T_{\infty}-T_{\text {ind }}}{2}\right)^{3}$, is the radiation coefficient which evaluates the intensity of convective heat transfer in comparison with radiative heat transfer.

Equation (3) expresses the relation between the indicated temperature, $T_{\text {ind }}$, (reply) and the process temperature, $T_{\text {proc }}$, (stimulus). The direct problem can be solved instantly through calculation of the output $\left(T_{\text {ind }}\right)$ from the known input $\left(T_{\text {proc }}\right)$.

Although encountering a solution to the corresponding inverse problem certainly is a difficult task because of its inherent ill-conditioned nature, it indicates that the solution of $T_{\text {proc }}$ from $T_{\text {ind }}$ is strongly affected by the presence of experimental errors from $T_{\text {ind }}$ measurements.

Then, by finite difference method, Eq. (3) can be discretized in time. Defining a time step $\Delta t$ and a backward discretization scheme with indices $n$ and $n-1$ indicates that the variable refers to times, respectively, $t=n \Delta t$ and $t=(n-1) \Delta t$, making possible to write:

$$
\frac{\tau}{\Delta t}\left(T_{\text {ind }, n}-T_{\text {ind }, n-1}\right)-\left(T_{\text {proc }, n}-T_{\text {ind }, n}\right)-\gamma_{n}\left(T_{\infty}-T_{\text {ind }, n}\right)=0
$$

Then, the direct and inverse problems are, respectively, obtained:

$$
\begin{gathered}
T_{\text {ind }, n}=\frac{1}{\frac{\tau_{n}}{\Delta t}+1+\gamma_{n}}\left(T_{\text {proc }, n}+\gamma_{n} T_{\infty}+\frac{\tau}{\Delta t} T_{\text {ind }, n-1}\right) \\
T_{\text {rec }, n}=T_{\text {proc }, n}=\frac{\tau}{\Delta t}\left(T_{\text {ind }, n}-T_{\text {ind }, n-1}\right)+T_{\text {ind }, n}-\gamma_{n}\left(T_{\infty}-T_{\text {ind }, n}\right)
\end{gathered}
$$

The reconstruction is effective, as shown in equation (Oliveira et al. 2006), so regularization methods must be adopted; embedded noise in the signal is amplified by the derivative 
of the Eq. (3). The temperature obtained from the inverse problem equation (6) shall be called reconstructed temperature $\left(T_{\mathrm{rec}}\right)$ and shall not be confused with the actual process temperature $\left(T_{\text {proc }}\right)$.

\section{Regularization method}

The method proposed in this paper is a regularization algorithm by cubic spline implemented in MATLAB ${ }^{\circledR}$ software. The smoothing has in its formulation the new function construction by spline function that minimizes the difference between the distances of given and smoothed points.

Considering $T_{\text {rec }}$ as the input data of a discrete model, the aim is to estimate a new sequence that minimizes Eq. (7).

$$
L=\lambda \sum_{i=1}^{n}\left(\frac{T_{\mathrm{rec}, i}-T_{\mathrm{reg}, i}}{T_{\mathrm{rec}, i}}\right)^{2}+(1-\lambda) \int_{t_{0}}^{t_{n}}\left(T_{\mathrm{reg}}^{\prime \prime}(t)\right)^{2} \mathrm{~d} t
$$

where $T_{\text {reg }}$ represents new points already smoothed and $\lambda$ is the parameter that controls the smoothing.

The smoothing by cubic splines method described in this paper was based on publications by Reinsch (1967), Pollock (1993) and Weinert (2009). Be the reconstruction temperature in the time $t_{i}$ provided by:

$$
T_{\text {rec }, i}=T_{\text {reg }, i}+\epsilon_{i}
$$

where $\epsilon$ is the difference (error) between the smoothed data $T_{\text {reg }}$ obtained by applying the regularization method and the original data $T_{\text {rec }}$ arising from the inverse problem equation (6). In this case, the reconstruction of $T_{\text {reg }}$ will be to build a function $S(t)$ that minimizes:

$$
L=\lambda \sum_{i=1}^{n}\left(\frac{T_{\mathrm{rec}, i}-T_{\mathrm{reg}, i}}{T_{\mathrm{rec}, i}}\right)^{2}+(1-\lambda) \int_{t_{0}}^{t_{n}}\left(S^{\prime \prime}(t)\right)^{2} \mathrm{~d} t
$$

being $n$ the number of points. The posterior term of Eq. (9) can be expressed as a sum of the second derivatives of each of the intervals; therefore

$$
\int_{t_{0}}^{t_{n}}\left(S^{\prime \prime}(t)\right)^{2} \mathrm{~d} t=\sum_{i=0}^{n-1} \int_{t_{i}}^{t_{i+1}}\left(S^{\prime \prime}(t)\right)^{2} \mathrm{~d} t
$$

Each spline is composed of cubic segments, then the second derivative at any interval is a linear function, with the independent term $2 b_{i}$ in $t_{i}, 2 b_{i+1}$ in $t_{i+1}$ and $H_{i}=t_{i+1}-t_{i}$, so the Eq. (10) becomes

$$
\int_{t_{0}}^{t_{n}}\left(S^{\prime \prime}(t)\right)^{2} \mathrm{~d} t=4 \int_{0}^{h_{i}}\left(b_{i}\left(1-\frac{t}{H_{i}}\right)+b_{i+1} \frac{t}{H_{i}}\right)^{2} \mathrm{~d} t
$$

Solving Eq. (11) provides the following:

$$
4 \int_{0}^{h_{i}}\left(b_{i}\left(1-\frac{t}{H_{i}}\right)+b_{i+1} \frac{t}{H_{i}}\right)^{2} \mathrm{~d} t=\frac{4 H_{i}}{3}\left(b_{i}^{2}+b_{i} b_{i+1}+b_{i+1}^{2}\right)
$$


It is possible to rewrite the function $S(t)$ and $S^{\prime \prime}(t)$ dependent on variables $b_{i}$ and $T_{\text {reg, } i}$.

Considering the coordinates $\left(t_{i}, T_{\text {reg }, i}\right),\left(t_{i+1}, T_{\text {reg }, i+1}\right)$ and implied conditions:

$$
\begin{gathered}
S_{i}\left(t_{i}\right)=T_{\text {reg }, i} \\
S_{i}\left(t_{i+1}\right)=T_{\text {reg, } i+1} \\
S_{i}^{\prime \prime}\left(t_{i}\right)=2 b_{i} \\
S_{i}^{\prime \prime}\left(t_{i+1}\right)=2 b_{i+1}
\end{gathered}
$$

Equation (13) is an identity and Eq. (14) is an equality. Equation (14) can be opened as a cubic function, thus:

$$
a_{i} H_{i}^{3}+b_{i} H_{i}^{2}+c_{i} H_{i}+T_{\text {reg }, i}=T_{\text {reg }, i+1}
$$

Isolation of $c_{i}$ term from Eq. (17) provides the following:

$$
c_{i}=\frac{T_{\mathrm{reg}, i+1}-T_{\mathrm{reg}, i}}{H_{i}}-a_{i} H_{i}^{2}+b_{i} H_{i}
$$

Equation (15) is an identity and Eq. (16) is an equality. Equation (16) can be opened as the second derivative of Eq. (17) as:

$$
2 b_{i+1}=6 a_{i} H_{i}+2 b_{i}
$$

Isolation of $a_{i}$ term from Eq. (19) provides the following:

$$
a_{i}=\frac{b_{i+1}-b_{i}}{3 H_{i}}
$$

Substitution of Eq. (20) into (18) provides that:

$$
c_{i}=\frac{T_{\text {reg }, i+1}-T_{\text {reg }, i}}{H_{i}}-\frac{1}{3}\left(b_{i+1}-2 b_{i}\right) H_{i}
$$

Derivation of Eq. (17) and subsequent isolation $c_{i}$ provides the following:

$$
3 a_{i-1} H_{i-1}^{2}+2 b_{i-1} H_{i-1}+c_{i-1}=c_{i}
$$

Rearrangement of Eqs. (21) and (22) appears as the following:

$$
b_{i-1} H_{i-1}+2 b_{i}\left(H_{i-1}+H_{i}\right)+b_{i-1} H_{i}=\frac{3}{H_{i}}\left(T_{\text {reg }, i+1}-T_{\text {reg }, i}\right)-\frac{3}{H_{i-1}}\left(T_{\text {reg }, i}-T_{\text {reg }, i-1}\right)
$$

In accordance with the condition of natural spline, the first and the last terms of vector $b$ are null, that is, $b_{0}=b_{n}=0$, with $i=1,2, \ldots, n-1$. A matrix system can be written from Eq. (23): 


$$
\begin{aligned}
& \underbrace{\left[\begin{array}{cccccc}
p_{2} & H_{2} & 0 & \ldots & 0 & 0 \\
H_{2} & p_{2} & H_{3} & \ldots & 0 & 0 \\
0 & H_{3} & p_{4} & \ldots & 0 & 0 \\
\vdots & \vdots & \vdots & \ldots & \vdots & \vdots \\
0 & 0 & 0 & \ldots & p_{n-2} & H_{n-2} \\
0 & 0 & 0 & \ldots & H_{n-2} & p_{n-1}
\end{array}\right]}_{[A]} \underbrace{\left[\begin{array}{c}
b_{1} \\
b_{2} \\
b_{3} \\
\vdots \\
b_{n-2} \\
b_{n-1}
\end{array}\right]}_{\{b\}} \\
& =\underbrace{\left[\begin{array}{ccccccc}
r_{1} & f_{2} & r_{2} & 0 & \ldots & 0 & 0 \\
0 & r_{2} & f_{3} & r_{3} & \ldots & 0 & 0 \\
\vdots & \vdots & \vdots & \vdots & \ldots & \vdots & \vdots \\
0 & 0 & 0 & 0 & \ldots & r_{n-2} & 0 \\
0 & 0 & 0 & 0 & \ldots & f_{n-1} & r_{n-1}
\end{array}\right]}_{[F]} \underbrace{\left[\begin{array}{c}
T_{\mathrm{reg}, 1} \\
T_{\mathrm{reg}, 2} \\
\vdots \\
T_{\text {reg, }, n-1} \\
T_{\text {reg }, n}
\end{array}\right]}_{\left\{T_{\mathrm{reg}}\right\}}
\end{aligned}
$$

where

$$
\begin{gathered}
H_{i}=t_{i+1}-t_{i} \\
p_{i}=2\left(H_{i-1}+H_{i}\right) \\
r_{i}=\frac{3}{H_{i}} \\
f_{i}=-\left(\frac{3}{H_{i-1}}+\frac{3}{H_{i}}\right)=-\left(r_{i-1}+r_{i}\right)
\end{gathered}
$$

Rewriting Eq. (24) in matrix notation:

$$
[A]\{b\}=[F]\left\{T_{\text {reg }}\right\}
$$

Using the same notation in Eq. (12) and replacing in Eq. (19), we obtain

$$
L=\lambda\left\{T_{\text {rec }}\right\}^{-1}\left(\left\{T_{\text {rec }}\right\}-\left\{T_{\text {reg }}\right\}\right)^{2}+\frac{2}{3}(1-\lambda)[A]\{b\}^{2}
$$

Isolation of $\{b\}$ from Eq. (29) provides:

$$
\{b\}=[A]^{-1}[F]\left\{T_{\text {reg }}\right\}
$$

Equation (31) contains two unknowns, $\{b\}$ and $\left\{T_{\text {reg }}\right\}$ and, thus, the substitution of an unknown by a known variable enables solution of the system. Substituting Eq. (31) into Eq. (30), the terms from Eq. (32) become dependent on $\left\{T_{\text {reg }}\right\}$ :

$$
L\left(\left\{T_{\text {reg }}\right\}\right)=\lambda\left\{T_{\text {rec }}\right\}^{-1}\left(\left\{T_{\text {rec }}\right\}-\left\{T_{\text {reg }}\right\}\right)^{2}+\frac{2}{3}(1-\lambda)[A]^{-1}[F]^{2}\left\{T_{\text {reg }}\right\}^{2}
$$

Optimization of $\left\{T_{\text {reg }}\right\}$ values minimizes Eq. (32) by differentiation with the respective $\left\{T_{\text {reg }}\right\}$ and the result equals zero, thus

$$
-2 \lambda\left\{T_{\text {rec }}\right\}^{-1}\left(\left\{T_{\text {rec }}\right\}-\left\{T_{\text {reg }}\right\}\right)+\frac{4}{3}(1-\lambda)[A]^{-1}[F]^{2}\left\{T_{\text {reg }}\right\}=0
$$


Rearrangement of Eq. (33) and subsequent substitution into Eq. (31) provide

$$
\lambda\left\{T_{\text {rec }}\right\}^{-1}\left(\left\{T_{\text {rec }}\right\}-\left\{T_{\text {reg }}\right\}\right)=\frac{2}{3}(1-\lambda)[F]\{b\}
$$

Multiplying Eq. (34) by $\lambda^{-1}[F]\left\{T_{\text {rec }}\right\}$ and rearrangement through its identity, Eq. (29) becomes

$$
\underbrace{\left([A]+\mu[F]^{2}\right)}_{[E]}\{b\}=\underbrace{[F]\left\{T_{\mathrm{rec}}\right\}}_{[D]}
$$

where

$$
\mu=\frac{2(1-\lambda)}{3 \lambda}
$$

Rewriting Eq. (35) and isolating vector $\{b\}$ provides

$$
\{b\}=[E]^{-1}[D]
$$

With the value for vector $\{b\}$, the smoothing vector $\left\{T_{\text {reg }}\right\}$ can be obtained by

$$
\left\{T_{\text {reg }}\right\}=\left\{T_{\text {rec }}\right\}-\mu\{b\}[F]
$$

Thus, the data $\left\{T_{\mathrm{rec}}\right\}$ will be smoothed by the following steps:

1. Assemble matrices $[A]$ and $[F]$ from Eq. (24) with values from Eqs. (25), (26), (27) and (28);

2. Assemble matrices $[E]$ and $[D]$ according to Eq. (35);

3. Determine vector $\{b\}$ from Eq. (37);

4. Calculate the regularization vector $\left\{T_{\text {reg }}\right\}$ with the previously chosen modified smoothing parameter $\mu$.

\section{Spline cubic properties}

In regularization by cubic spline, $\lambda$ is the smoothing control parameter. The domain ranges from zero to one, where $\lambda=0$ considers that only the smoothing is taken into consideration, and the spline function $S(t)$ becomes a linear regression. At the other extreme, where $\lambda=1$ the main concern is the proximity to the points, which forms a spline interpolation and the function crosses the points accurately (Pollock 1993). The anticipated choice of the lambda parameter allows a more flexible method for adjusting the different sets of data that may require the technique. Besides the traditional regularization described in Sect. 3, it is possible to add a weight to the formulation and emphasize the smoothing. A weighted smoothing method was proposed by Green and Silverman (1994) and was based on Reisch's algorithm (Reinsch 1967).

The modified method requires an input vector $w$ with elements $w_{i}$, strictly positive, introduced in Eq. (9):

$$
L=\lambda \sum_{i=1}^{n} w_{i}\left(\frac{T_{\mathrm{rec}, i}-T_{\mathrm{reg}, i}}{T_{\mathrm{rec}, i}}\right)^{2}+(1-\lambda) \int_{t_{0}}^{t_{n}}\left(S^{\prime \prime}(t)\right)^{2} \mathrm{~d} t
$$

The method implementation consists of a matrix $[W]$ of $\{w\}$ vector elements, which is incorporated directly into the cubic spline smoothing algorithm in Eq. (35):

$$
\underbrace{\left([A]+\mu[F]^{2}\right)[W]^{-1}}_{[E]}\{b\}=\underbrace{[F]\left\{T_{\mathrm{rec}}\right\}[W]}_{[D]}
$$


And thus add weight to the regularization. However, this work proposes a simpler and more efficient addition of weight than described by Green and Silverman (1994), reducing to zero the amount of weight matrix and incorporating the penalty in itself regularization matrix constructed during the process.

This alternative weighting must be an integer natural non-zero number, and the higher the constant the higher is the gains on smoothing. The weight is introduced on the right side of Eq. (23):

$$
\begin{aligned}
& b_{i-1} H_{i-1}+2 b_{i}\left(H_{i-1}+H_{i}\right)+b_{i-1} H_{i} \\
& \quad=\left(\frac{3}{H_{i}}\left(T_{\text {reg }, i+1}-T_{\text {reg }, i}\right)-\frac{3}{H_{i-1}}\left(T_{\text {reg }, i}-T_{\text {reg }, i-1}\right)\right) w
\end{aligned}
$$

which corresponds to the elements from matrix $[F]$. In algorithm, the weight's constant multiplies the terms $\frac{3}{H_{i}}$ and $\frac{3}{H_{i-1}}$ in Eq. (27):

$$
r_{i}=w \frac{3}{H_{i}}
$$

\section{Results and discussion}

Figure 1 presents a test simulation, consisting of a sine wave $\left(T_{\text {proc }}\right)$ representing the real process temperature in which the signal captured by a thermocouple without encapsulation is used as the method validation reference. The second signal simulation is the temperature indicated $\left(T_{\text {ind }}\right)$, captured by a encapsulated thermocouple and, therefore, presents delays and noise inherent from the process. The inverse problem equation (6), Sect. 2, obtained from $T_{\text {ind }}$ reveals the reconstructed temperature $T_{\text {rec }}$. Observation of $T_{\text {rec }}$ values shows high-frequency components' amplification, where most of the noise is contained. Thus, application of cubic spline regularization on $T_{\text {rec }}$ values (with value $\lambda=0.5$ ) provides the regularized temperature $\left(T_{\text {reg }}\right)$. Finally, $T_{\text {rec }}$ smoothed by weighted cubic spline method, according to Eqs. (41) and (42) in Sect. 4 ( $w=2$ and $\lambda=0.5)$, provides the weighted regularized temperature ( $\left.T_{\text {regpond }}\right)$.

Comparative analysis between standard regularization method $\left(T_{\text {reg }}\right)$ and the function available by MATLAB $^{\circledR}$ (Matlab) was conducted (Fig. 2), as well as another between weighted regularization method (Tregpond) [Green and Silverman (1994) $\left(T_{\text {regGS }}\right.$ ), (Fig. 3)] - the parameters used are the same as the Fig. 1 results. It is noticeable from both

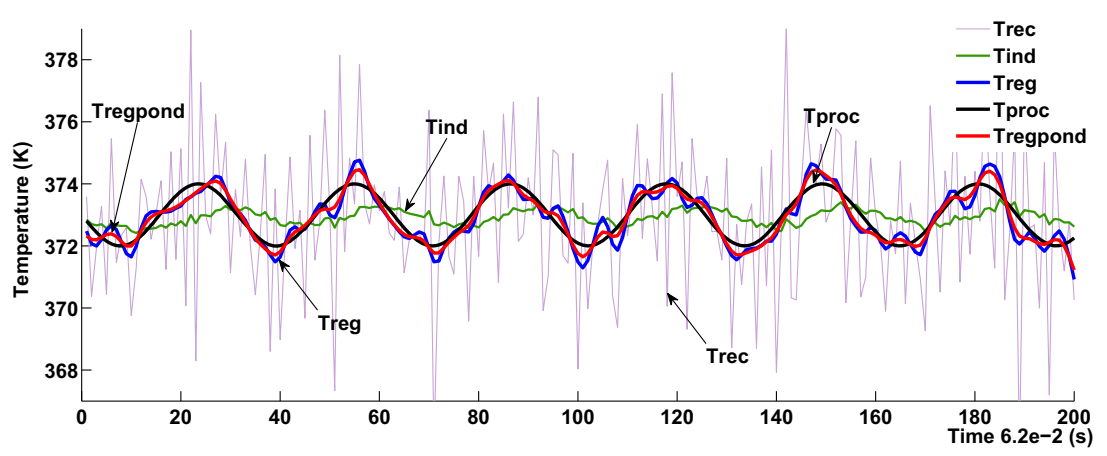

Fig. 1 Reconstruction and regularization of the simulated signal 


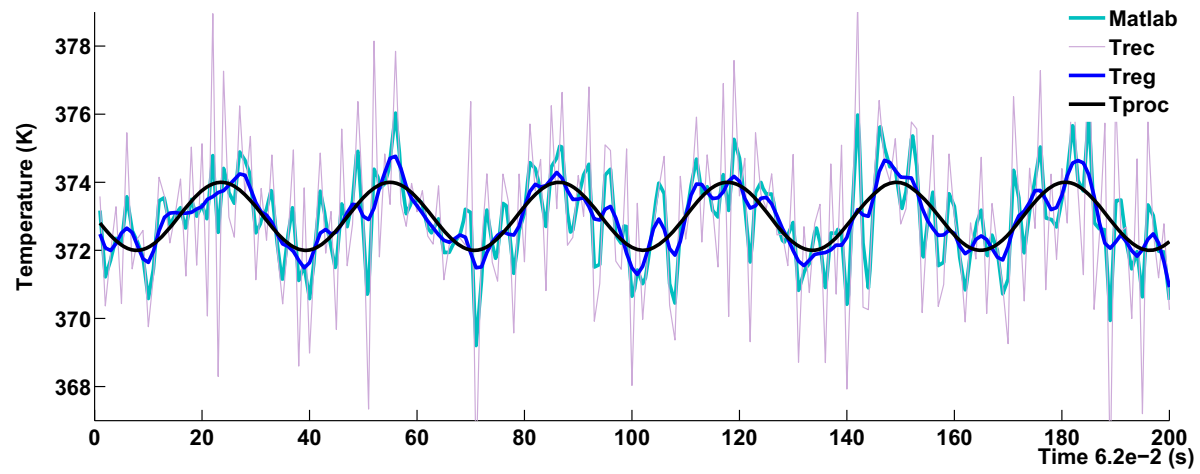

Fig. 2 Simulated signal standard regularization

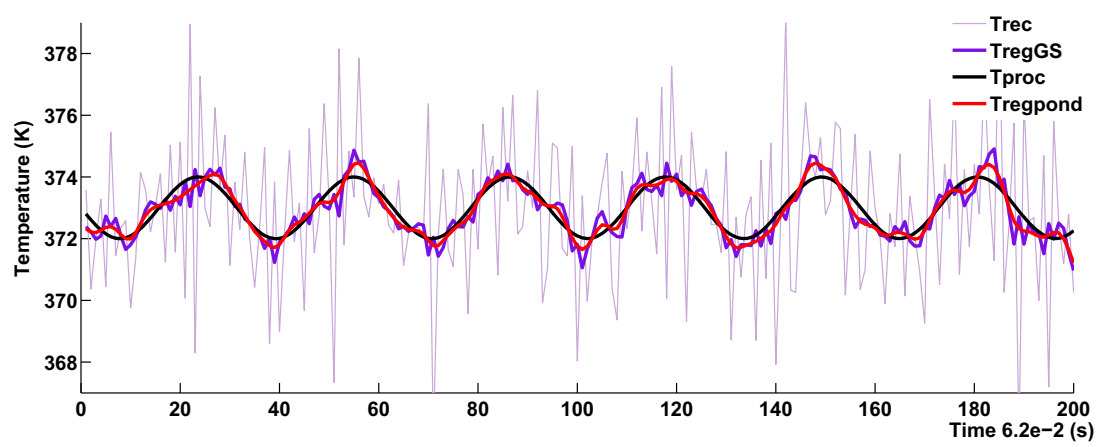

Fig. 3 Weighted form regularization of the simulated signal

plots that the temperature signals $T_{\text {reg }}$ and $T_{\text {regpond }}$ are closer to the $T_{\text {proc }}$ than the signals $T_{\text {regGS }}$ and Matlab.

The results were analyzed after regularization between the process temperature $T_{\text {proc }}$ and regularized without penalty increase $T_{\text {reg }}$, the Green and Silverman's weighting (Green and Silverman 1994), $T_{\text {regGS }}$, and the alternative weighting, $T_{\text {regpond }}$, temperatures through the root mean square error (RMSE):

$$
E=\sqrt{\frac{\sum_{i=1}^{n}\left(T_{\mathrm{proc}, i}-T_{\mathrm{reg}, i}\right)^{2}}{n}}
$$

The RMSE value provides an absolute agreement estimate between the process and the regularized temperatures.

The weighted regularization may vary the formula's parameter as $0 \geq \lambda \geq 1$ and weight $w$ added to Eq. (42). Implementation of an optimized search system for parameter and weight choice, based on RMSE, generated the values in Table 1 .

The $T_{\text {regpond }}$ plot for the lowest RMSE $=1.658$, with $w=7$ and $\lambda=0.6$, can be seen in Fig. 4. The plot contains discrepancy analysis of the implement regularization $\left(T_{\text {reg }}\right)$, with $\lambda=0.5$. It is noticeable that the weighted regularized curve approaches $T_{\text {proc }}$ almost perfectly, which is the main objective, to reconstruct the real process temperature. 
Table 1 Root mean square error (RMSE) and parameter variations

\begin{tabular}{llllllllll}
\hline $\mathrm{w} \backslash \mathrm{t}$ & 0.1 & 0.2 & 0.3 & 0.4 & 0.5 & 0.6 & 0.7 & 0.8 & 0.9 \\
\hline 1 & 2.169 & 2.761 & 3.208 & 3.620 & 4.047 & 4.566 & 5.219 & 6.151 & 7.926 \\
2 & 1.66 & 1.837 & 2.144 & 2.454 & 2.761 & 3.093 & 3.489 & 4.047 & 5.163 \\
3 & 2.047 & 1.660 & 1.733 & 1.921 & 2.169 & 2.454 & 2.79 & 3.24 & 4.047 \\
4 & 2.792 & 1.861 & 1.662 & 1.698 & 1.837 & 2.059 & 2.358 & 2.761 & 3.455 \\
5 & 3.553 & 2.279 & 1.804 & 1.663 & 1.69 & 1.819 & 2.056 & 2.424 & 3.059 \\
6 & 4.238 & 2.792 & 2.081 & 1.764 & 1.66 & 1.698 & 1.854 & 2.169 & 2.761 \\
7 & 4.829 & 3.305 & 2.454 & 1.963 & 1.724 & 1.658 & 1.733 & 1.975 & 2.526 \\
8 & 5.329 & 3.791 & 2.849 & 2.229 & 1.861 & 1.682 & 1.674 & 1.837 & 2.332 \\
9 & 5.749 & 4.238 & 3.241 & 2.539 & 2.047 & 1.764 & 1.658 & 1.744 & 2.169 \\
10 & 6.100 & 4.643 & 3.619 & 2.856 & 2.279 & 1.889 & 1.683 & 1.69 & 2.034 \\
20 & 7.733 & 6.925 & 6.158 & 5.403 & 4.643 & 3.867 & 3.074 & 2.279 & 1.693 \\
30 & 8.224 & 7.733 & 7.237 & 6.704 & 6.1 & 5.403 & 4.573 & 3.553 & 2.279 \\
\hline
\end{tabular}

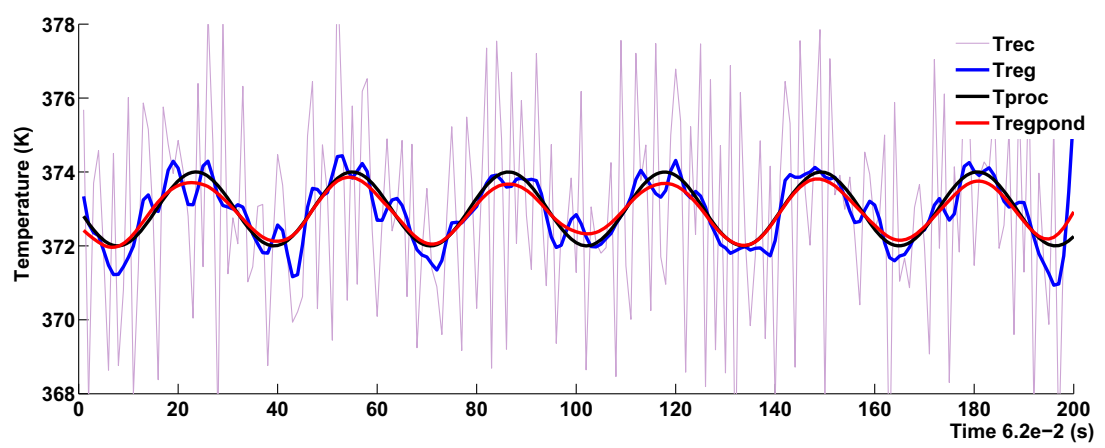

Fig. 4 Comparison of standard smoothing and weighting described in the work

Table 2 Mean process times for weight and absolute error

\begin{tabular}{llrr}
\hline Amount of points & 200 & \multicolumn{1}{c}{4000} & \multicolumn{1}{c}{10,000} \\
\hline Time $T_{\text {regpond }}(\mathrm{s})$ & 0.020 & 124.356 & 2158.600 \\
Error $T_{\text {proc }} / T_{\text {regpond }}$ & 4.408 & 18.385 & 28.904 \\
Time $T_{\text {regGS }}(\mathrm{s})$ & 0.029 & 188.810 & 3296.400 \\
Error $T_{\text {proc }} / T_{\text {regGS }}$ & 4.972 & 20.950 & 33.226 \\
\hline
\end{tabular}

The alternative weighted regularization was also compared with that described in Green and Silverman (1994) as the efficiency reference and using the same weight values $w=2$ and $\lambda=0.5$.

To verify if the weighted regularization method described herein is more efficient than the one proposed by Green and Silverman (1994), signals of different sizes (200, 400 and 10000 points) were simulated. The algorithm related to the time of execution as well as the absolute error is shown in Table 2. The weighted regularization method showed the most promising 
results, either regarding the time of execution or the absolute error, because the implemented algorithm does not require any additional matrices, besides smoothing the signal effectively.

\section{Experimental tests}

To prove the efficiency of the method and weighting, experimental tests were conducted with hot air jets from a dryer Black \& Decker Inc., model SC831B and $1500 \mathrm{~W}$ power. Being the distance between the air jet and thermocouples $s_{j}=0.15 \mathrm{~m}$, the distance between the two thermocouples $s=0.002 \mathrm{~m}$ and encapsulation is composed of long-lasting nail polish.

The data acquisition board used to capture the temperatures was made by National Instruments Corporation and is the type Rack with connection USB CDAQ-9171 and analog input module for thermocouples NI 9211 with four channels $80 \mathrm{mV}$, sampling $14 \mathrm{~S} / \mathrm{s}$ and 24 bits of resolution. For the measurement of temperature, thermocouples type $\mathrm{K}$ was used. The board was connected to the computer, as data acquisition and storage measurements were recorded with software NI-DAQMX and software LabVIEW ${ }^{\mathrm{TM}}$ (version 11.0) and subsequent evaluation of these data collected.

Figure 5 represents the complete experimental set layout with hot air source, thermocouples connected with data acquisition board and subsequent data acquisition and storage with LabVIEW $^{\mathrm{TM}}$.

As shown in Fig. 6, composed of the experimental data from Fig. 5, the smoothing provided by the software MATLAB ${ }^{\circledR}$ was able to reduce $T_{\text {rec }}$ peaks, but it was far from the $T_{\text {proc }}$ target. Although the weighted regularization proposed by Green and Silverman (1994) $\left(T_{\text {regGS }}\right)$ is

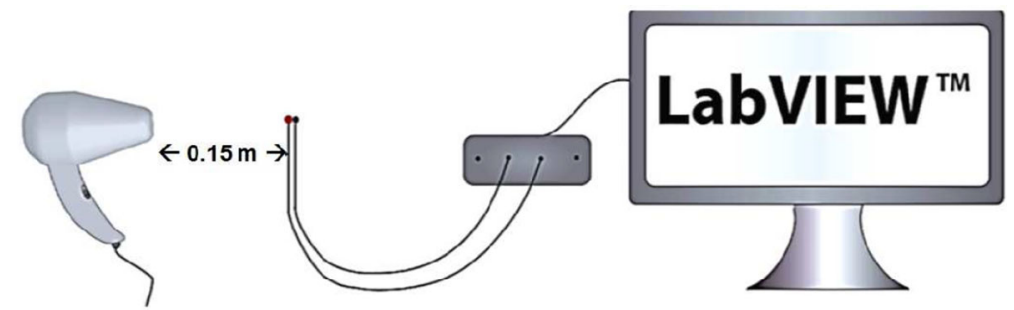

Fig. 5 Experimental work design

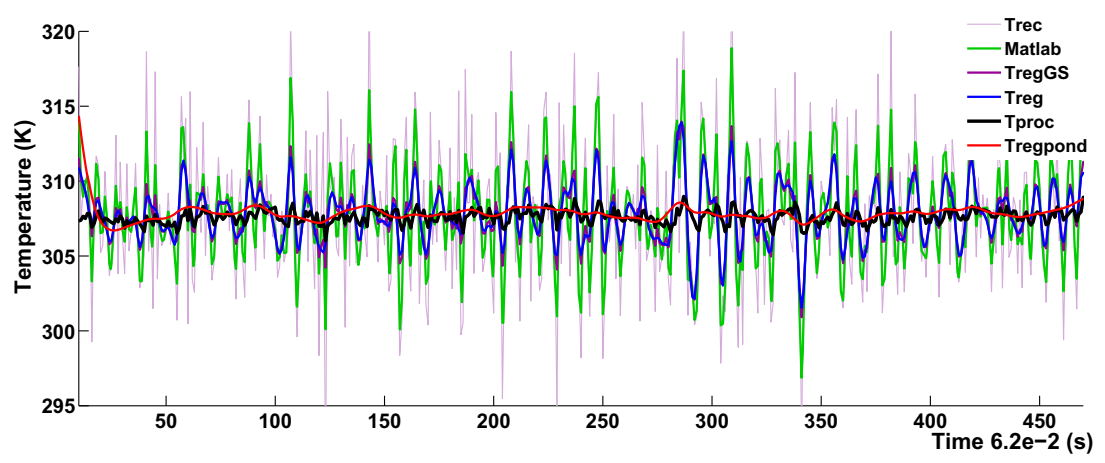

Fig. 6 Experimental signal smoothing 


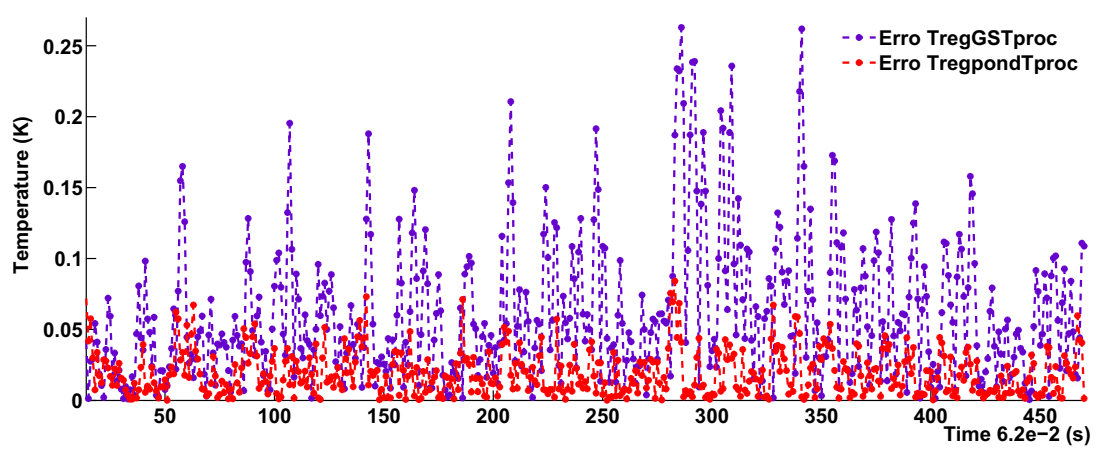

Fig. 7 RMSE of the weighting by Green and Silverman (1994) and the new weighting proposed at work

able to produce a stronger smoothing when compared to MATLAB ${ }^{\circledR}$ (Matlab), the standard smoothing $\left(T_{\text {reg }}\right)$ implemented in this work has proved to be even better. To find the best parameter and weight for the weighted regularization proposed herein, the search system that was used follows the model exhibited in Table 1. Therefore, the lowest RMSE was obtained ( $w=9$ and $\lambda=0.2$ ) and shows that the proposed regularization was able to smooth as well as keep the $T_{\text {regpond }}$ in the same temperature range as $T_{\text {proc }}$. The addition and search for the best parameter and weights, the weighted regularization $T_{\text {regpond }}$ was able to smooth and maintain the same temperature range as $T_{\text {proc }}$. The choice for the best parameter and weight were made through the construction of a table following the model Table 1 and the lowest RMSE indicated the values searched being $w=9$ and $\lambda=0.2$.

Figure 7 shows a point to point difference between the two weighted smoothing form $T_{\text {regpond }}$ and $T_{\text {regGS }}$, using the RMSE value between the smoothing and the reference temperature $T_{\text {proc }}$. As can be observed, the error of the proposed weighted smoothing method is smaller than the described by Green and Silverman (1994), proving the efficiency of cubic spline smoothing method implemented in this work.

\section{Conclusion}

This work proposed an alternative to the regularization method of cubic spline weighted form applied in solving inverse thermal problems. The study involved the capture of a thermal delayed and attenuated signal that has been reconstructed using the inverse model of transduction equation. However, the reconstructed temperature differs significantly from the real values, because of all the uncertainties associated with inverse problems requiring a filter to soften the noise and delay the reconstructed signal. The method used was smoothed by cubic spline and its weighted form. The ability to select the best technique was through comparisons between standard regularization provided by MATLAB ${ }^{\circledR}$ and implemented in Sect. 3, and the weighted regularization provided by Green and Silverman (1994) and the new weighting method described in this paper (Sect. 4). The presented alternative weighting method is simpler and more effective, because it does not compromise the algorithm execution time and is easy to comprehend by relying on a single nonzero positive value, and without need for the construction and manipulation of large matrices. The simulated and experimental examples presented in the work were able to undoubtedly illustrate the new method's efficiency. 


\section{References}

Alifanov OM (1977) Determination of heat loads from a solution of the nonlinear inverse problem. High Temp 15(3):498-504

Alifanov OM (1994) Inverse heat transfer problems. Springer, New York

Beck JV, Blackwell B, St Clair CR (1985) Inverse heat conduction: Ill-posed problems. Wiley, New York

Green P, Silverman B (1994) Nonparametric regression and generalized linear models: a roughness penalty approach. Chapman \& Hall/CRC Press, Boca Raton

Hadamard J (1923) Lectures on Cauchys problem in linear differential equations. In: Technical report. Yale University, New Haven

Jakubowska M (2011) Signal processing in electrochemistry. Electroanalysis 23:553-572

Kabala ZJ (1997) Inverse thermal problems. Am Sci 85(3):288

Kaipio JP, Fox C (2011) The Bayesian framework for inverse problems in heat transfer, vol 32

Kim HJ, Kim NK, Kwak JS (2006) Heat flux distribution model by sequential algorithm of inverse heat transfer determining workpiece temperature in creep feed grinding. Int J Mach Tool Man 46:2086-2093

Oliveira J, Santos J, Seleghim P Jr (2006) Inverse measurement method for detecting bubbles in a fluidized bed reactor-toward the development of an intelligent temperature sensor. Powder Technol 169:123-135

Orlande HRB (2011) Inverse heat transfer problems, vol 32. doi:10.1080/01457632.2011.525128

Özisik H, Orlande HRB (2000) Inverse heat transfer: fundamentals and applications. Taylor \& Francis, New York

Park HM, Chung OY, Lee JH (1999) On the solution of inverse heat transfer problem using the Karhunen-loève Galerkin method. Int J Heat Mass Transf 42(1):127-142

Pollock DSG (1993) Smoothing with cubic splines. In: Technical report

Reinsch CH (1967) Smoothing by spline function. Numer Math 10:177-183

Tikhonov AN, Arsenin VY (1977) Solution of Ill—posed problems. Winston \& Sons, Washington

Weinert HL (2009) A fast compact algorithm for cubic spline smoothing. Comput Stat Data Anal 53:932-940 